\title{
Sub-THz technology for dynamic nuclear polarization in nuclear magnetic resonance (DNP NMR): transverse confinement of microwave propagation through heterogeneous solid DNP samples
}

\author{
Frank Engelke ${ }^{1}$, Armin Purea ${ }^{1}$, Christian Reiter ${ }^{1}$, Fabien Aussenac $^{2}$ \\ ${ }^{1}$ Bruker Biospin GmbH, Rheinstetten, Germany, frank.engelke@bruker.com \\ ${ }^{2}$ Bruker Biospin S.A., Wissembourg, France
}

Passive microwave components for DNP NMR, the resulting spatial microwave field distribution at the sample site, the sample structure, the molecular structure, and DNP spin physics are closely linked to each other. We study specific examples of low-temperature (100 K) DNP samples, characterized by a high degree of spatial heterogeneity on a sub-mm scale. We report on our work in progress investi-gating the local microwave propagation modified through transverse confinement in DNP samples with possible consequences for the DNP spin transfer mechanism.

DNP NMR [1] on solids samples, in particular, under magic-angle spinning (MAS) has become an extremely useful technique in solid-state NMR to enhance the weak NMR signal by one or two orders of magnitude [2-4]. Application examples range from the study of biomacromolecules [5-6] to material samples like zeolites or mesoporous silica with small molecules or catalytic compounds adsorbed at the inner surfaces of the mesoporous material [7-9].

The mechanism by which DNP allows to enhance the nuclear spin polarization signals is based on a coherent spin polarization transfer in a strong static external magnetic field from unpaired electron spins in paramagnetic centers (e.g., radicals) to nuclear spins under irradiation of electron spin-resonant microwaves. A particular DNP mechanism working at high magnetic fields (e.g., 9$18 \mathrm{~T}$ ) requires biradicals in the sample, now with two unpaired electron spins coupled by dipole-dipole interaction. Together with a nearby situated nuclear spin they form a three-spin system that gives rise to the DNP cross effect [10-11] being specifically efficient in DNP MAS NMR.

Probes for MAS NMR capable of performing DNP experiments [12] inherit various traits from the subsystems composing them, which may lead to conflicting or challenging probe hardware boundary conditions. The sample is packed into a zirconia or sapphire MAS rotor spinning at a speed of ca. $10 \mathrm{kHz}$, driven by an airbearing microturbine. Tightly wound around the MAS rotor there is an NMR rf solenoidal coil with interturn spacings such that the radially (to the rotor axis) incident $\mathrm{HE}_{11}$ or $\mathrm{TEM}_{00}$ Gaussian microwave beam can irradiate the sample inside the rotor. A schematic view is shown in Fig. 1. The MAS system and the sample are kept at temperatures around $100 \ldots 200 \mathrm{~K}$ in order to keep the spin relaxation time $T_{1 \mathrm{e}}$ of the unpaired electron spins in the biradicals at large enough values (micro to milliseconds) to maintain the electron spin polarization or coherences during the DNP experiment.

For a variety of DNP samples an additional complication appears whenever the sample is heterogeneous on a sub-mm scale. Examples are powders of mesoporous or nanoporous samples, layered samples of oriented biomembranes, or, e.g., simply powders with a grain size distribution on the order of 100 to $400 \mu \mathrm{m}$, impregnated by a solvent (e.g., tetra-chloroethane, TCE) and dissolved in it some biradical (AMUPol, TEKPol, and others) frozen at low temperature. It has been shown experimentally, that the strongly inhomogeneous microwave field in such samples leads to quite high DNP enhancement factors, in the case of dielectric particle powders impregnated with frozen biradical solutions one even may observe DNP enhancement factors being two- to three-fold higher [13] than those in homogeneous samples with well defined periodic microwave field distributions.

The heterogeneity of such samples strongly affects the microwave propagation, because in the case of frozen impregnated powders there is a random distribution of spatial regions with differing dielectric constants, giving rise to multiple reflections and dif- fraction of local microwave beamlets. It is well known from first principles, that for charge-free regions Gauss' law $\operatorname{div} \mathbf{E}=0$ applies. Close to surfaces, e.g., of the dielectric particles, and inside cavities or pores of sub-wavelength dimensions this leads to local microwave electric fields $\mathbf{E}$ and local Poynting vectors $\mathbf{P}$ that are not orthogonal anymore, because the presence of surfaces and pore walls imposes transverse confinement to the local field such that longitudinal $\mathbf{E}$ field components arise. Fig. 2 represents an example of dielectric particles (sapphire, $\varepsilon=9.6$ ) embedded in frozen TCE $(\varepsilon=2.5)$ and illustrates the simulated (by CST Microwave Studio ${ }^{\circledR}$, www.cst.com) spatial distribution of the angle between the local Poynting vector and the local electric field in a rotor axial plane parallel to the incident microwave beam. The resulting complex pattern reflects the randomness inherent in the powder distribution of sapphire particles and causes an equally randomized distribution of local microwave electric and magnetic field amplitudes and directions which directly relates to the performance of DNP experiments. Only the microwave magnetic field vector component orthogonal to the external static magnetic field couples to the electron spin and causes electron spin transitions that lead to saturation (equal occupation of Zeeman spin levels).

In conclusion, we suggest that the simulated complex spatial distribution of amplitudes and local angular dependence of Poynting and electric field vectors appears as a result of the transverse confinement [14-15] in strongly heterogeneous DNP samples. It gives rise, apart from the powder distribution of EPR $g$ tensors, to a statistical distribution of microwave magnetic field vectors representing the randomness of particle or pore distribution in the sample. Yet we do not know the details of 


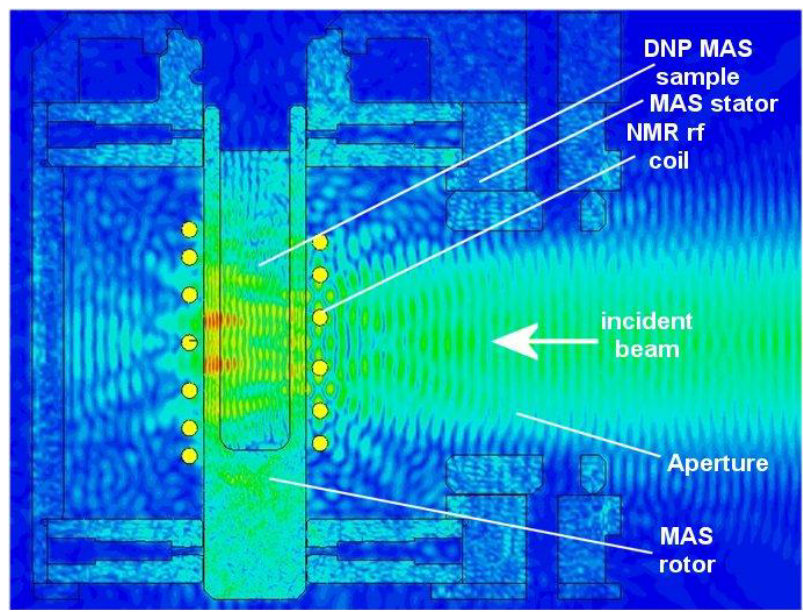

Fig. 1. Schematic view of an DNP MAS NMR setup with the rotor containing the sample (here supposed to be homogeneous), surrounded by an solenoidal coil tuned to the desired NMR frequencies, and the incident $\mathrm{mm}$ wave beam (here at 395 $\mathrm{GHz}$ ) being diffracted and partly reflected by the coil, rotor, and the sample

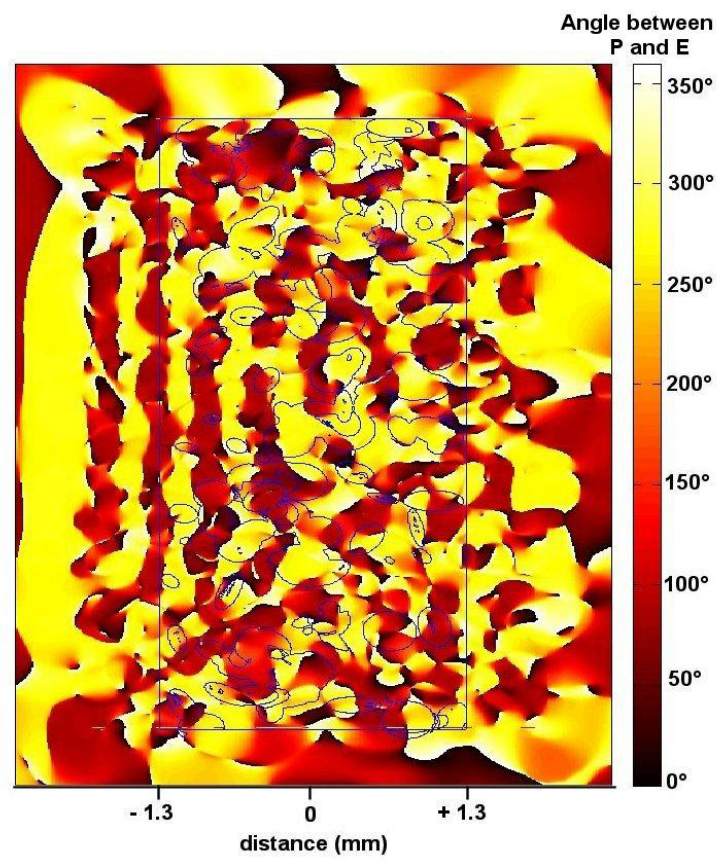

Fig. 2. Spatial distribution in a rotor axial plane of the angle between local Poyning vector $\mathbf{P}$ and local electric field vector $\mathbf{E}$ in a sample of sapphire powder (grain size distribution 100 $400 \mu \mathrm{m}$ ) impregnated with tetrachloroethane / TEKPol biradical, frozen in a solid matrix at $\mathrm{T}=100 \mathrm{~K}$. The linearly polarized Gaussian microwave beam, waist $3 \mathrm{~mm}$, frequency $263 \mathrm{GHz}$, is incident from the left side. In the simulation, only the rotor and the heterogeneous sample material is present. $3 \mathrm{D}$ voxel resolution $10 \mu \mathrm{m}, 5 \times 10^{7}$ mesh cells of the field distribution relevant for EPR spin excitation and DNP, but take that as a forthcoming task: to quantify the impact of the microwave field distribution on the coherent DNP spin dynamics. Further it needs to be shown in a quantitative manner that the transverse confinement in typical heterogeneous DNP samples may lead, jointly with spin diffusion in such structures, to an improvement of the enhancement factor in DNP mechanisms like the DNP cross effect.

\section{References}

1. Carver, T.R., Slichter, C.P. Polarization of nuclear spins in metals // Phys. Rev. 1953. V. 92. P. 212.

2. Becerra L.R., et al. Dynamic nuclear polarization with a cyclotron resonance maser at 5 T // Phys. Rev. Lett. 1993. V. 71. P. 3561.

3. Gerfen, G. J., et al. High frequency $(140 \mathrm{GHz})$ dynamic nuclear polarization: polarization transfer to a solute in frozen aqueous solution // J. Chem. Phys.1995. V. 102. P. 9494.

4. Rosay, M., et al., Solid-state dynamic nuclear polarization at $263 \mathrm{GHz}$ : spectrometer design and experimental results // Phys. Chem. Chem. Phys. 2010. V. 12. P. 5850.

5. Akbey, U., Oschkinat, $H$. Structural biology applications of solid state MAS DNP NMR // J. Magn. Reson. 2016. V. 269. P. 213.

6. Debelouchina, G. T., et al. Dynamic nuclear polarization-enhanced solid-state NMR spectroscopy of GNNQQNY nanocrystals and amyloid fibrils // Phys. Chem. Chem. Phys. 2010. V. 12, N. 22. P. 5911.

7. Lesage, A., et al. Surface enhanced NMR spectroscopy by dynamic nuclear polarization // J. Am. Chem. Soc. 2010. V. 132, N. 44. P. 15459.

8. Lafon, $O$., et al. Mesoporous Silica Nanoparticles Loaded with Surfactant: Low Temperature Magic Angle Spinning 13C and 29Si NMR Enhanced by Dynamic Nuclear Polarization // J. Phys. Chem. C. 2013. V. 117, N. 3. P. 1375.

9. Perras, F. A., et al. Natural Abundance 17O DNP: TwoDimensional and Surface-Enhanced NMR Spectroscopy // J. Am. Chem. Soc. 2015. V. 137. P. 8336.

10. Hovav, Y., et al. Theoretical aspects of dynamic nuclear polarization in the solid state - The cross effect // J. Magn. Reson. 2012. V. 214. P. 29.

11. Maly, T., et al. Dynamic nuclear polarization at high magnetic fields // J. Chem. Phys. 2008. V. 128, N. 5. P. 052211.

12. Rosay, M., et al. Instrumentation for solid-state dynamic nuclear polarization with magic angle spinning NMR // J. Magn. Reson. 2016. V. 264. P. 88.

13. Kubicki, D.J., et al. Amplifying dynamic nuclear polarization of frozen solutions by incorporating dielectric particles // J. Am. Chem. Soc. 2014. V. 136, N. 44. P. 15711.

14. Anderson, P.W. Absence of diffusion in certain random lattices // Phys. Rev. 1958. V. 109. P. 1492.

15. Cherroret, N., et al. Transverse confinement of waves in three-dimensional random media // Phys. Rev. E 2010. V. 82. P. 056603. 